\title{
Can We Use Placental Growth Factor-Based Tests to Assess Women With Suspected Pre-eclampsia?
}

\author{
Nevin Sağsöz $z^{*}$
}

$\mathrm{P}$ re-eclampsia is a major risk factor for maternal and perinatal mortality with an incidence of about $2-8 \%$. Its diagnosis is based on new-onset hypertension and raised urinary protein excretion after 20 weeks of gestation. In the absence of proteinuria, the diagnosis can be made with hypertension together with the accompanying signs or symptoms of significant endorgan dysfunction (1). Therefore, the diagnosis may be difficult and complicated in some cases.

Moreover, angiogenic factors are thought to play a key role in placental vascular development and the formation of pre-eclampsia. The balance shifts to the anti-angiogenic direction, especially in more severe and early-onset forms of pre-eclampsia. Although the release of some placental anti-angiogenic molecules such as soluble fms-like tyrosine kinase 1 (sFlt-1) and soluble endoglin (sEng) increases in maternal circulation, pro-angiogenic substances such as placental growth factor (PIGF) and vascular endothelial growth factor A (VEGF-A) demonstrate a decrease $(2,3)$.

In the last decade, various new and interesting studies have addressed this issue, particularly to assess women with suspected pre-eclampsia (4-6). For instance, Duhig et al. found that the adoption of PlGF testing as a diagnostic adjunct in women presenting with suspected pre-eclampsia would decrease the time for clinicians to make a diagnosis in women with suspected pre-eclampsia. They further indicated that this approach would reduce subsequent maternal or perinatal adverse outcomes (4). Similarly, McCarthy et al compared three commercially available placental growth factor-based tests in women with suspected preterm pre-eclampsia, including the DELFIA ${ }^{\circledR}$ Xpress PlGF 1-2-3 (PerkinElmer, Wallac Oy, Turku, Finland), the Triage ${ }^{\circledR}$ PlGF (Alere, Inc., San Diego, CA, USA), and the Elecsys ${ }^{\circledR}$ sFlt-1 to PlGF ratio (Roche Diagnostics GmbH, Mannheim, Germany) tests (5).

All these tests had similar performance in the prediction of the need for delivery within 14 days in women with suspected pre-eclampsia. Furthermore, these tests had high negative predictive values thus they could be used as 'rule-out' tests for pre-eclampsia (5). Moreover, the

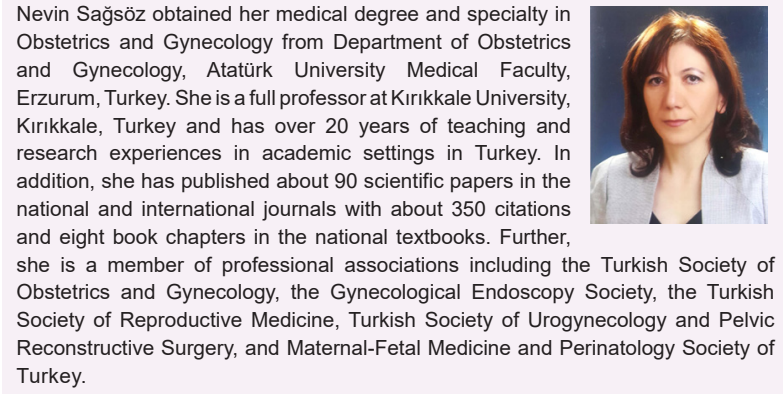

Triage ${ }^{\circledR}$ PlGF and the Elecsys ${ }^{\circledR}$ sFlt-1 to PlGF ratio tests were recommended in the The National Institute for Health and Care Excellence (NICE) guidance to help ruleout pre-eclampsia in women with suspected pre-eclampsia between 20 and 34 weeks plus six days of gestation. It was also emphasized that although these tests are not in the routine usage, they show promises in helping to diagnose (rule-in) pre-eclampsia in women demonstrating suspected pre-eclampsia between 20 and 34 weeks plus six days of gestation (6).

In conclusion, placental growth factor-based tests seem to be valuable for assessing women with suspected pre-eclampsia and can be recommended in the clinical management algorithms for the clinicians.

\section{Ethical Issues}

Not applicable.

\section{Conflict of Interests}

The author has no conflicts of interest to disclose.

\section{References}

1. ACOG Practice Bulletin No. 202: Gestational Hypertension and Preeclampsia. Obstet Gynecol. 2019;133(1):e1-e25. doi:10.1097/aog.0000000000003018

2. Dymara-Konopka W, Laskowska M, Blazewicz A. Angiogenic Imbalance as a Contributor of Preeclampsia. Curr Pharm Biotechnol. 2018;19(10):797-815. doi:10.2174 $/ 1389201019666180925115559$ 
3. Cim N, Kurdoglu M, Ege S, Yoruk I, Yaman G, Yildizhan R. An analysis on the roles of angiogenesis-related factors including serum vitamin $\mathrm{D}$, soluble endoglin (sEng), soluble fms-like tyrosine kinase 1 (sFlt1), and vascular endothelial growth factor (VEGF) in the diagnosis and severity of late-onset preeclampsia. J Matern Fetal Neonatal Med. 2017;30(13):1602-1607. doi:10.1080/14767058.2016.1 219986

4. Duhig KE, Myers J, Seed PT, et al. Placental growth factor testing to assess women with suspected pre-eclampsia: a multicentre, pragmatic, stepped-wedge cluster-randomised controlled trial. Lancet. 2019;393(10183):1807-1818. doi:10.1016/s0140-6736(18)33212-4
5. McCarthy FP, Gill C, Seed PT, Bramham K, Chappell LC, Shennan AH. Comparison of three commercially available placental growth factor-based tests in women with suspected preterm pre-eclampsia: the COMPARE study. Ultrasound Obstet Gynecol. 2019;53(1):62-67. doi:10.1002/ uog. 19051

6. National Institute for Health and Care Excellence (NICE). PlGF-based testing to help diagnose suspected preeclampsia (Triage PlGF test, Elecsys immunoassay sFlt-1/ PlGF ratio, DELFIA Xpress PlGF 1-2-3 test, and BRAHMS sFlt-1 Kryptor/BRAHMS PlGF plus Kryptor PE ratio). NICE; 2016.

Copyright (c) 2019 The Author(s); This is an open-access article distributed under the terms of the Creative Commons Attribution License (http://creativecommons.org/licenses/by/4.0), which permits unrestricted use, distribution, and reproduction in any medium, provided the original work is properly cited. 